\section{P208 Process Evaluation of the Smarter Lunchrooms RCT: Unique Application of the RE-AIM Framework}

Alisha Gaines, PhD, againes@cornell.edu, Cornell University, 342A MVR Hall, Ithaca, NY 14853;

T. Hill, MPH; L. Thomas, PhD; J. Dollahite, PhD, RD

Objective: To adapt the RE-AIM framework for use in process evaluation of the Smarter Lunchrooms (SL) RCT.

Design, Setting and Participants: The SL intervention aimed to improve student food selection using small, low cost environmental changes. During the past two years, SL was implemented in 25 New York state middle school cafeterias. Parallel process evaluation was conducted to assess effectiveness of intervention delivery and inform interpretation of results.

Outcome Measures and Analysis: Year one process evaluation methods were categorized according to dimensions of the RE-AIM framework (reach, effectiveness, adoption, implementation, and maintenance) in order to identify gaps and future directions.

Results: Reach was defined as the number of eligible middle schools participating in the intervention and the number of enrolled students in each school. Effectiveness is typically defined in terms of intervention outcomes; however, from a process evaluation perspective, it could also serve as an outlet for discussion of external influences expected to affect intervention outcomes, specifically sources of contamination and aspects of school cafeterias already aligned with the protocol. Adoption was defined as the number and percentage of cafeteria staff trained and their reported preparedness to initiate the intervention. Implementation was described as fidelity to each component of the intervention protocol, while maintenance was the extent of adherence beyond the intervention end date.

Conclusions and Implications: RE-AIM served as a valuable framework for designing a process evaluation for an environmental intervention. This modified model may be useful for future researchers undertaking similar planning or evaluation, and for those seeking to operationalize RE-AIM dimensions to meet unique needs of an environmental intervention.

Funding: NIFA.

\section{P209 Influences That Drive Americans to Commence, Continue, and Cease Food or Beverage Consumption}

Rachel Paul, MS, RD, rachel.paul@tc.columbia.edu, Teachers College, Columbia University, 525 W. 120th Street, New York, NY 10027; M. Smith-Edge, MS, RD, LD, $F A D A$, International Food Information Council Foundation; M. Greenblum, MS, RDN; B. O'Meara, Southern Methodist University

Objective: Overweight and obesity are primarily issues of energy imbalance. While food choice is critical, eating too often and too much are also contributing factors. The current study aimed to examine influences pertaining to the commencement, continuation, and cessation of eating and drinking occasions.

Design, Setting and Participants: Semi-structured focus group interviews with twelve groups involving 40 participants (41\% male; $64.3 \%$ white; $19.0 \%$ black; $11.9 \%$ Hispanic) were conducted in three urban areas across the country with young adults (18-29y), parents and working adults (30-45y), and older adults (46-65y).

Outcome Measures and Analysis: Videotaped focus groups were coded and analyzed to identify themes. Social cognitive theory built the theoretical framework for data analysis.

Results: Cues to action, positive outcome expectations, perceived barriers, and affective attitudes were theorybased determinants uniquely identified for starting, continuing, and/or stopping food and beverage consumption, often differing by life-stage group. Certain eating occasions were planned or unplanned throughout the day; breakfast planning was rote with little consideration of taste, while lunch planning encompassed who to eat with, where to eat, and what to eat. Hunger cues were present or anticipated; many began eating to satisfy hunger, while others began and continued eating in order to avoid being unproductive, irritable, and hungry in the future. An individual's environment also influenced starting or stopping eating, such as while traveling or throughout a sports event.

Conclusions and Implications: Not only what we eat, but how often and how much we eat at various eating occasions is influential to energy balance. More research examining these factors as targets for behavior change is warranted.

Funding: International Food Information Council Foundation.

\section{P210 Strategies to Improve Diet Quality Among Children With Autism Spectrum Disorders (ASDs): A Qualitative Study}

Rachel Paul, MS, RD, rachel.paul@tc.columbia.edu, Teachers College, Columbia University, 525 W. 120th Street, New York, NY 10027; J. Michalski, BS, Columbia University Medical Center; R. Hand, BS, Teachers College, Columbia University; H. L. Gray, PhD, RD

Objective: As many as 80 percent of children with ASDs are reported as picky eaters. Consequently, many suffer from issues ranging from suboptimal nutrient intake to obesity. Although different strategies for practitioners have been described, specific recommendations to improve diet quality remain undetermined. The aim of this study was to delineate such strategies for children with ASDs.

Design, Setting and Participants: Medical professionals $(\mathrm{N}=10)$ including registered dietitians, psychologists, and behavioral, occupational, and speech therapists, who worked with feeding issues, participated in semi-structured interviews from September 2014 through January 2015. 